\title{
Mean X-ray attenuation of salivary calculi computed from microtomography data
}

Nolasco, P*, Coelho, P. V. ${ }^{* *}$, Alves de Matos, A.P..**, Maurício, A.***, Pereira, M.F.C. ${ }^{* * *}$, Sanches, J.M.R. ${ }^{* * * * * *}$, Carvalho, P.A."

* ICEMS, Instituto Superior Técnico, University of Lisbon, Av. Rovisco Pais, 1049-001 Lisboa, Portugal

** Maxillofacial Surgery, Centro Hospitalar de Lisboa Central, Hospital S. José, R. José António Serrano, 1150-199 Lisboa, Portugal

**** CiiEM - Centro de Investigação Interdisciplinar Egas Moniz, Campus Universitário, Quinta da Granja , Monte de Caparica, 2829-511 Caparica, Portugal

***** CEGPIST/CERENA, Department of Civil Engineering, Architecture and Georesources, Instituto Superior Técnico, University of Lisbon, Av. Rovisco Pais, 1049-001 Lisboa, Portugal

****** ISR, Department of Bioengineering, Instituto Superior Técnico, University of Lisbon, Av. Rovisco Pais, 1049-001 Lisboa, Portugal

Email: pedro.nolasco@ist.utl.pt

Salivary calculi, or sialoliths, have an estimated clinical prevalence of 0.12 to $0.45 \%$ [1]. Long-term obstruction of the ducts by sialoliths can lead to atrophy of the salivary glands with concomitant ceasing of the secretory function and ultimately fibrosis [2]. Shock wave lithotripsy (SWL) is a non-invasive therapeutic technique that can be used to eliminate salivary calculi [3], however the method has had a less than desirable success rate [3,4,5], which may be justified by the sialoliths' fraction of organic mater [6]. Earlier studies have found a correlation between the calculi size and degree of mineralization with the outcome of SWL treatment $[7,8]$. It is therefore essential to systematically characterize salivary calculi in terms of these parameters. The present work aims to characterize the volume and degree of mineralization of salivary calculi through X-ray micro computed tomography $(\mu \mathrm{CT})$.

Figure 1 shows a microradiograph of a submandibular sialolith (a) and a median longitudinal reconstructed cross-section obtained from $\mu \mathrm{CT}$ data (b) where brighter regions correspond to higher mineralization and dark regions represent essentially organic matter. Table 2 presents the average volume and X-ray attenuation of submandibular calculi $\left(\mathrm{S}_{\mathrm{i}}\right)$ together with the corresponding $\mathrm{Ca}+\mathrm{Mg}$ fraction determined by induced couple atomic emission spectroscopy. Although the results present high variability, there is a correlation between the amount of $\mathrm{Ca}+\mathrm{Mg}$ and the degree of mineralization as measured by the mean X-ray attenuation. These results indicate that X-ray tomography may be used to identify the patients with calculi susceptible to ultra-sound shockwaves.

The authors acknowledge financial support of the Portuguese Foundation for Science and Technology through PTDC/SAU-ENB/111941/2009, PEst-OE/CTM-UI0084/2011 and PEst-OE/CTM-UI0098/2011 grants. Communication submitted to the Microscopy at the Frontiers of Science 2013 congress in Tarragona, Spain.

[1] Escudier, M., Controversies in the Management of Salivary Gland Disease, Oxford University 251-259, 2013.

[2] Williams, M.F., Otolaryngologic Clinics of North America, 32, 819-834, 1999.

[3] Schmitz, S. et al., Journal of Laryngology \& Otolology, 122, 65-71, 2008.

[4] Iro H. et al., Laryngoscope, 119, 263-268, 2009. 
[5] Capaccio, P., Laryngoscope, 114, 1069-1073, 2004.

[6] Nolasco, P. et al., Microscopy and Microanalysis, 19, 1-14, 2013.

[7] Escudier, M.P, et al, The Laryngoscope, 120, 1545-1549, 2010.

[8] Mcain, P.D, et al, Reviews in urology, 15, 49-60, 2013.

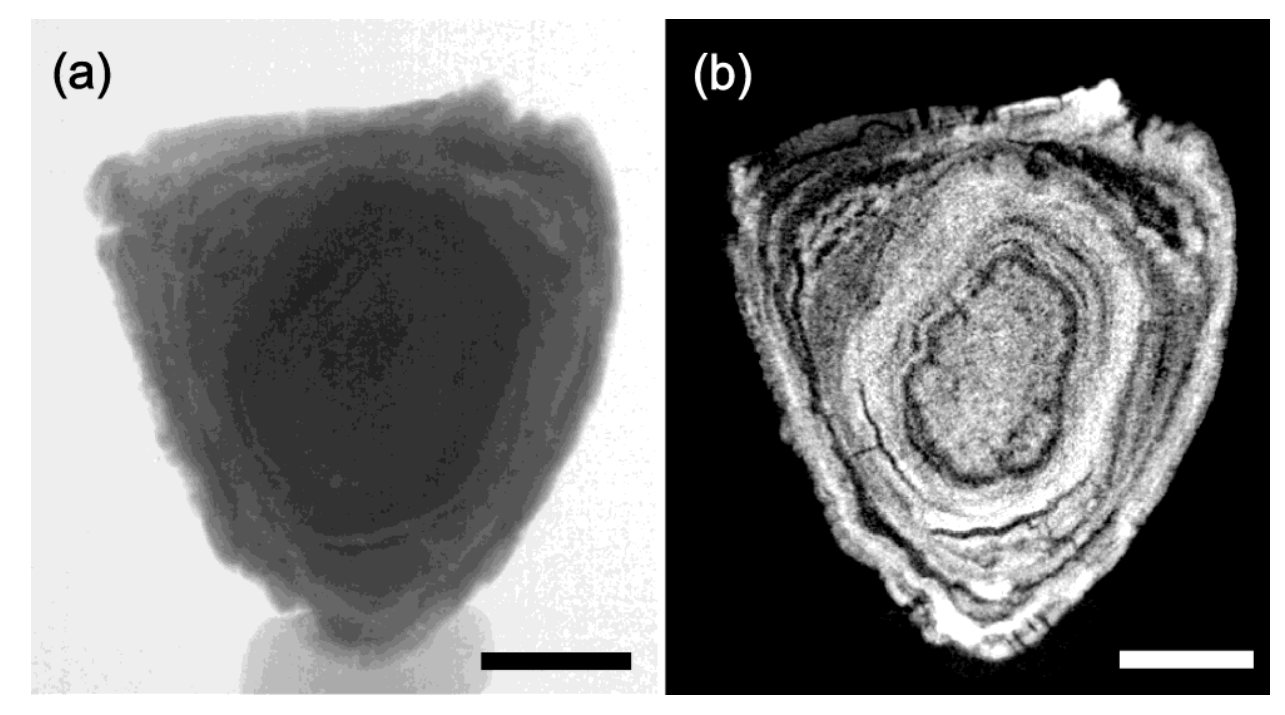

Figure 1 - (a) Microradiograph of a submandibular sialolith and (b) a median longitudinal reconstructed cross-section obtained from $\mu \mathrm{CT}$ data. The scale bars correspond to $2 \mathrm{~mm}$.

Table 1 - Mean attenuation and volume determined from $\mu \mathrm{CT}$ data, and $\mathrm{Ca}+\mathrm{Mg}$ fraction measured by induced couple atomic emission spectroscopy.

\begin{tabular}{|c|c|c|c|}
\hline Sample & $\begin{array}{c}\text { Mean attenuation } \\
\left(10^{3} \mathrm{~m}^{-1}\right)\end{array}$ & $\begin{array}{c}\text { Volume } \\
\left(10^{-9} \mathrm{~m}^{3}\right)\end{array}$ & $\begin{array}{c}\text { Fraction of Ca+Mg } \\
(\text { wt \%) }\end{array}$ \\
\hline $\mathrm{S}_{1}$ & 0.017 & 227.82 & 8.86 \\
$\mathrm{~S}_{2}$ & 0.025 & 61.26 & 11.71 \\
$\mathrm{~S}_{3}$ & 0.033 & 384.64 & 18.54 \\
$\mathrm{~S}_{4}$ & 0.045 & 455.66 & 23.96 \\
\hline
\end{tabular}

